\title{
A Comparative Study of Wireless Protocols: Bluetooth, UWB, ZigBee, and Wi-Fi
}

\author{
Jin-Shyan Lee, Yu-Wei Su, and Chung-Chou Shen \\ Information \& Communications Research Labs \\ Industrial Technology Research Institute (ITRI) \\ Hsinchu, Taiwan \\ jinshyan_lee@itri.org.tw
}

\begin{abstract}
Bluetooth (over IEEE 802.15.1), ultra-wideband (UWB, over IEEE 802.15.3), ZigBee (over IEEE 802.15.4), and Wi-Fi (over IEEE 802.11) are four protocol standards for shortrange wireless communications with low power consumption. From an application point of view, Bluetooth is intended for a cordless mouse, keyboard, and hands-free headset, UWB is oriented to high-bandwidth multimedia links, ZigBee is designed for reliable wirelessly networked monitoring and control networks, while Wi-Fi is directed at computer-to-computer connections as an extension or substitution of cabled networks. In this paper, we provide a study of these popular wireless communication standards, evaluating their main features and behaviors in terms of various metrics, including the transmission time, data coding efficiency, complexity, and power consumption. It is believed that the comparison presented in this paper would benefit application engineers in selecting an appropriate protocol.
\end{abstract}

Index Terms - Wireless protocols, Bluetooth, ultra-wideband (UWB), ZigBee, Wi-Fi, short-range communications.

\section{INTRODUCTION}

In the past decades, factory automation has been developed worldwide into a very attractive research area. It incorporates different modern disciplines including communication, information, computer, control, sensor, and actuator engineering in an integrated way, leading to new solutions, better performance and complete systems. One of the increasingly important components in factory automation is the industrial communication [1]. For interconnection purposes, a factory automation system can be combined with various sensors, controllers, and heterogeneous machines using a common message specification. Many different network types have been promoted for use on a shop floor, including control area network (CAN), Process fieldbus (Profibus), Modbus, and so on. However, how to select a suitable network standard for a particular application is a critical issue to the industrial engineers. Lain et al. [2] evaluated the Ethernet (carrier sense multiple access with collision detection, CSMA/CD bus), ControlNet (token-passing bus), and DeviceNet (CSMA with arbitration on message priority, CSMA/AMP bus) for networked control applications. After a detailed discussion of the medium access control (MAC) sublayer protocol for each network, they studied the key parameters of the corresponding network when used in a control situation, including network utilization and time delays.
On the other hand, for accessing networks and services without cables, wireless communications is a fast-growing technology to provide the flexibility and mobility [3]. Obviously, reducing the cable restriction is one of the benefits of wireless with respect to cabled devices. Other benefits include the dynamic network formation, low cost, and easy deployment. General speaking, the short-range wireless scene is currently held by four protocols: the Bluetooth, and UWB, ZigBee, and $\mathrm{Wi}-\mathrm{Fi}$, which are corresponding to the IEEE 802.15.1, 802.15.3, 802.15.4, and $802.11 \mathrm{a} / \mathrm{b} / \mathrm{g}$ standards, respectively. IEEE defines the physical (PHY) and MAC layers for wireless communications over an action range around 10-100 meters. For Bluetooth and Wi-Fi, Ferro and Potorti [4] compared their main features and behaviors in terms of various metrics, including capacity, network topology, security, quality of service support, and power consumption. In [5], Wang et al. compared the MAC of IEEE 802.11e and IEEE 802.15.3. Their results showed that the throughput difference between them is quite small. In addition, the power management of 802.15.3 is easier than that of 802.11e. For ZigBee and Bluetooth, Baker [6] studied their strengths and weaknesses for industrial applications, and claimed that ZigBee over 802.15.4 protocol can meet a wider variety of real industrial needs than Bluetooth due to its long-term battery operation, greater useful range, flexibility in a number of dimensions, and reliability of the mesh networking architecture.

In this paper, after an overview of the mentioned four shortrange wireless protocols, we attempt to make a preliminary comparison of them and then specifically study their transmission time, data coding efficiency, protocol complexity, and power consumption. The rest of this paper is organized as follows. Section II briefly introduces the wireless protocols including Bluetooth, UWB, ZigBee, and Wi-Fi. Next, a comprehensive evaluation of them is described in Section III. Then, in Section IV, the complexity and power consumption are compared based on IEEE standards and commercial offthe-shelf wireless products, respectively. Finally, Section V concludes this paper.

\section{WIRELESS PROTOCOLS}

This section introduces the Bluetooth, UWB, ZigBee, and Wi-Fi protocols, which corresponds to the IEEE 802.15.1, 
802.15.3, 802.15.4, and $802.11 \mathrm{a} / \mathrm{b} / \mathrm{g}$ standards, respectively. The IEEE defines only the PHY and MAC layers in its standards. For each protocol, separate alliances of companies worked to develop specifications covering the network, security and application profile layers so that the commercial potential of the standards could be realized.

The material presented in this section is widely available in the literature. Hence, the major goal of this paper is not to contribute to research in the area of wireless standards, but to present a comparison of the four main short-range wireless networks.

\section{A. Bluetooth over IEEE 802.15.1}

Bluetooth, also known as the IEEE 802.15.1 standard is based on a wireless radio system designed for short-range and cheap devices to replace cables for computer peripherals, such as mice, keyboards, joysticks, and printers. This range of applications is known as wireless personal area network (WPAN). Two connectivity topologies are defined in Bluetooth: the piconet and scatternet. A piconet is a WPAN formed by a Bluetooth device serving as a master in the piconet and one or more Bluetooth devices serving as slaves. A frequency-hopping channel based on the address of the master defines each piconet. All devices participating in communications in a given piconet are synchronized using the clock of the master. Slaves communicate only with their master in a point-to-point fashion under the control of the master. The master's transmissions may be either point-to-point or point-tomultipoint. Also, besides in an active mode, a slave device can be in the parked or standby modes so as to reduce power consumptions. A scatternet is a collection of operational Bluetooth piconets overlapping in time and space. Two piconets can be connected to form a scatternet. A Bluetooth device may participate in several piconets at the same time, thus allowing for the possibility that information could flow beyond the coverage area of the single piconet. A device in a scatternet could be a slave in several piconets, but master in only one of them.

\section{B. UWB over IEEE 802.15 .3}

UWB has recently attracted much attention as an indoor short-range high-speed wireless communication. [7]. One of the most exciting characteristics of UWB is that its bandwidth is over $110 \mathrm{Mbps}$ (up to $480 \mathrm{Mbps}$ ) which can satisfy most of the multimedia applications such as audio and video delivery in home networking and it can also act as a wireless cable replacement of high speed serial bus such as USB 2.0 and IEEE 1394. Following the United States and the Federal Communications Commission (FCC) frequency allocation for UWB in February 2002, the Electronic Communications Committee (ECC TG3) is progressing in the elaboration of a regulation for the UWB technology in Europe. From an implementation point of view, several solutions have been developed in order to use the UWB technology in compliance with the FCC's regulatory requirements. Among the existing PHY solutions, in IEEE 802.15 Task Group 3a (TG3a), multiband orthogonal frequency-division multiplexing (MB-OFDM), a carrier-based system dividing UWB bandwidth to sub-bands, and direct-sequence UWB (DS-UWB), an impulse-based system that multiplies an input bit with the spreading code and transmits the data by modulating the element of the symbol with a short pulse have been proposed by the WiMedia Alliance and the UWB Forum, respectively. The TG3a was established in January 2003 to define an alternative PHY layer of 802.15.3. However, after three years of a jammed process in IEEE 802.15.3a, supporters of both proposals, MB-OFDM and DS-UWB, supported the shut down of the IEEE 802.15.3a task group without conclusion in January 2006. On the other hand, IEEE 802.15.3b, the amendment to the 802.15.3 MAC sublayer has been approved and released in March 2006.

\section{ZigBee over IEEE 802.15.4}

ZigBee over IEEE 802.15.4, defines specifications for lowrate WPAN (LR-WPAN) for supporting simple devices that consume minimal power and typically operate in the personal operating space (POS) of $10 \mathrm{~m}$. ZigBee provides self-organized, multi-hop, and reliable mesh networking with long battery lifetime [8-9]. Two different device types can participate in an LR-WPAN network: a full-function device (FFD) and a reduced-function device (RFD). The FFD can operate in three modes serving as a PAN coordinator, a coordinator, or a device. An FFD can talk to RFDs or other FFDs, while an RFD can talk only to an FFD. An RFD is intended for applications that are extremely simple, such as a light switch or a passive infrared sensor. They do not have the need to send large amounts of data and may only associate with a single FFD at a time. Consequently, the RFD can be implemented using minimal resources and memory capacity. After an FFD is activated for the first time, it may establish its own network and become the PAN coordinator. All star networks operate independently from all other star networks currently in operation. This is achieved by choosing a PAN identifier, which is not currently used by any other network within the radio sphere of influence. Once the PAN identifier is chosen, the PAN coordinator can allow other devices to join its network. An RFD may connect to a cluster tree network as a leave node at the end of a branch, because it may only associate with one FFD at a time. Any of the FFDs may act as a coordinator and provide synchronization services to other devices or other coordinators. Only one of these coordinators can be the overall PAN coordinator, which may have greater computational resources than any other device in the PAN.

\section{Wi-Fi over IEEE $802.11 \mathrm{a} / \mathrm{b} / \mathrm{g}$}

Wireless fidelity (Wi-Fi) includes IEEE $802.11 \mathrm{a} / \mathrm{b} / \mathrm{g}$ standards for wireless local area networks (WLAN). It allows users to surf the Internet at broadband speeds when connected to an access point (AP) or in ad hoc mode. The IEEE 802.11 architecture consists of several components that interact to provide a wireless LAN that supports station mobility transparently to upper layers. The basic cell of an IEEE 802.11 LAN is called a basic service set (BSS), which is a set of mobile or fixed stations. If a station moves out of its BSS, it can no longer directly communicate with other members of the BSS. Based on the BSS, IEEE 802.11 employs the independent basic service set (IBSS) and extended service set (ESS) 
network configurations. As shown in Fig. 1, the IBSS operation is possible when IEEE 802.11 stations are able to communicate directly without any AP. Because this type of IEEE 802.11 LAN is often formed without pre-planning, for only as long as the LAN is needed, this type of operation is often referred to as an ad hoc network. Instead of existing independently, a BSS may also form a component of an extended form of network that is built with multiple BSSs. The architectural component used to interconnect BSSs is the distribution system (DS). The DS with APs allow IEEE 802.11 to create an ESS network of arbitrary size and complexity. This type of operation is often referred to as an infrastructure network.

\section{COMPARATIVE STUDY}

Table I summarizes the main differences among the four protocols. Each protocol is based on an IEEE standard. Obviously, UWB and Wi-Fi provide a higher data rate, while Bluetooth and ZigBee give a lower one. In general, the Bluetooth, UWB, and ZigBee are intended for WPAN communication (about $10 \mathrm{~m}$ ), while Wi-Fi is oriented to
WLAN (about 100m). However, ZigBee can also reach $100 \mathrm{~m}$ in some applications.

FCC power spectral density emission limit for UWB emitters operating in the UWB band is $-41.3 \mathrm{dBm} / \mathrm{Mhz}$. This is the same limit that applies to unintentional emitters in the UWB band, the so called Part 15 limit. The nominal transmission power is $0 \mathrm{dBm}$ for both Bluetooth and ZigBee, and $20 \mathrm{dBm}$ for $\mathrm{Wi}-\mathrm{Fi}$.

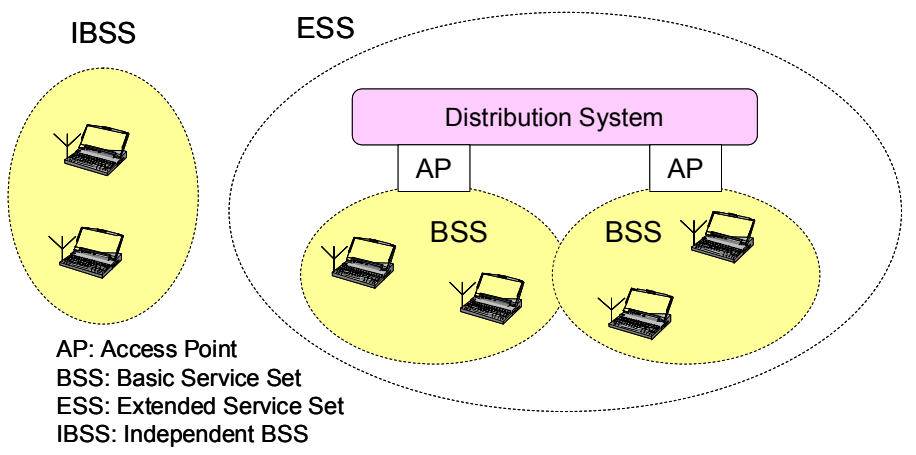

Fig. 1. IBSS and ESS configurations of Wi-Fi networks.

TABLE I

COMPARISON OF THE BLUETOOTH, UWB, ZigBeE, AND Wi-Fi PROTOCOLS

\begin{tabular}{|c|c|c|c|c|}
\hline Standard & Bluetooth & UWB & ZigBee & Wi-Fi \\
\hline IEEE spec. & 802.15 .1 & $802.15 .3 a$ * & 802.15 .4 & $802.11 \mathrm{a} / \mathrm{b} / \mathrm{g}$ \\
\hline Frequency band & $2.4 \mathrm{GHz}$ & $3.1-10.6 \mathrm{GHz}$ & $868 / 915 \mathrm{MHz} ; 2.4 \mathrm{GHz}$ & $2.4 \mathrm{GHz} ; 5 \mathrm{GHz}$ \\
\hline Max signal rate & $1 \mathrm{Mb} / \mathrm{s}$ & $110 \mathrm{Mb} / \mathrm{s}$ & $250 \mathrm{~Kb} / \mathrm{s}$ & $54 \mathrm{Mb} / \mathrm{s}$ \\
\hline Nominal range & $10 \mathrm{~m}$ & $10 \mathrm{~m}$ & $10-100 m$ & $100 \mathrm{~m}$ \\
\hline Nominal TX power & $0-10 \mathrm{dBm}$ & $-41.3 \mathrm{dBm} / \mathrm{MHz}$ & $(-25)-0 \mathrm{dBm}$ & $15-20 \mathrm{dBm}$ \\
\hline Number of RF channels & 79 & $(1-15)$ & $1 / 10 ; 16$ & $14(2.4 \mathrm{GHz})$ \\
\hline Channel bandwidth & $1 \mathrm{MHz}$ & $500 \mathrm{MHz}-7.5 \mathrm{GHz}$ & $0.3 / 0.6 \mathrm{MHz} ; 2 \mathrm{MHz}$ & $22 \mathrm{MHz}$ \\
\hline Modulation type & GFSK & BPSK, QPSK & BPSK (+ ASK), O-QPSK & $\begin{array}{c}\text { BPSK, QPSK } \\
\text { COFDM, CCK, M-QAM }\end{array}$ \\
\hline Spreading & FHSS & DS-UWB, MB-OFDM & DSSS & DSSS, CCK, OFDM \\
\hline Coexistence mechanism & Adaptive freq. hopping & Adaptive freq. hopping & Dynamic freq. selection & $\begin{array}{c}\text { Dynamic freq. selection, } \\
\text { transmit power control } \\
(802.11 \mathrm{~h})\end{array}$ \\
\hline Basic cell & Piconet & Piconet & Star & BSS \\
\hline Extension of the basic cell & Scatternet & Peer-to-peer & Cluster tree, Mesh & ESS \\
\hline Max number of cell nodes & 8 & 8 & $>65000$ & 2007 \\
\hline Encryption & E0 stream cipher & $\begin{array}{l}\text { AES block cipher } \\
\text { (CTR, counter mode) }\end{array}$ & $\begin{array}{l}\text { AES block cipher } \\
\text { (CTR, counter mode) }\end{array}$ & $\begin{array}{l}\text { RC4 stream cipher } \\
\text { (WEP), } \\
\text { AES block cipher }\end{array}$ \\
\hline Authentication & Shared secret & CBC-MAC (CCM) & CBC-MAC (ext. of CCM) & WPA2 (802.11i) \\
\hline Data protection & 16-bit CRC & 32-bit CRC & 16-bit CRC & 32-bit CRC \\
\hline \multicolumn{5}{|c|}{$\begin{array}{l}\text { * Unapproved draft. } \\
\text { - Acronyms: ASK (amplitude shift keying), GFSK (Gaussian frequency SK), BPSK/QPSK (binary/quardrature phase SK), O-QPSK (offset-QPSK), OFDM } \\
\text { (orthogonal frequency division multiplexing), COFDM (coded OFDM), MB-OFDM (multiband OFDM), M-QAM (M-ary quadrature amplitude modulation), CCK } \\
\text { (complementary code keying), FHSS/DSSS (frequency hopping/direct sequence spread spectrum), BSS/ESS (basic/extended service set), AES (advanced } \\
\text { encryption standard), WEP (wired equivalent privacy), WPA (Wi-Fi protected access), CBC-MAC (cipher block chaining message authentication code), CCM } \\
\text { (CTR with CBC-MAC), CRC (cyclic redundancy check). }\end{array}$} \\
\hline
\end{tabular}




\section{A. Radio Channels}

Bluetooth, ZigBee and Wi-Fi protocols have spread spectrum techniques in the $2.4 \mathrm{GHz}$ band, which is unlicensed in most countries and known as the industrial, scientific, and medical (ISM) band. Bluetooth uses frequency hopping (FHSS) with 79 channels and $1 \mathrm{MHz}$ bandwidth, while ZigBee uses direct sequence spread spectrum (DSSS) with 16 channels and $2 \mathrm{MHz}$ bandwidth. Wi-Fi uses DSSS (802.11), complementary code keying (CCK, 802.11b), or OFDM modulation $(802.11 \mathrm{a} / \mathrm{g})$ with $14 \mathrm{RF}$ channels $(11$ available in US, 13 in Europe, and just 1 in Japan) and $22 \mathrm{MHz}$ bandwidth. UWB uses the 3.1-10.6 GHz, with an unapproved and jammed 802.15.3a standard, of which two spreading techniques, DSUWB and MB-OFDM, are available.

\section{B. Coexistence Mechanism}

Since Bluetooth, ZigBee and Wi-Fi use the $2.4 \mathrm{GHz}$ band, the coexistence issue must be dealt with. Basically, Bluetooth and UWB provide adaptive frequency hopping to avoid channel collision, while ZigBee and Wi-Fi use dynamic frequency selection and transmission power control. IEEE 802.15.2 discussed the interference problem of Bluetooth and Wi-Fi. Also, Sikora and Groza [10] provided quantitative measurements of the coexistence issue for ZigBee, Bluetooth, Wi-Fi, and microwave ovens. Shuaib et al. [11] focused on quantifying potential interferences between Zigbee and IEEE $802.11 \mathrm{~g}$ by examining the impact on the throughput performance of IEEE 802.11g and Zigbee devices when coexisting within a particular environment. Moreover, Neelakanta and Dighe [12] presented a performance evaluation of Bluetooth and ZigBee collocated on an industrial floor for robust factory wireless communications.

\section{Network Size}

The maximum number of devices belonging to the network's building cell is 8 ( 7 slaves plus one master) for a Bluetooth and UWB piconet, over 65000 for a ZigBee star network, and 2007 for a structured Wi-Fi BSS. All the protocols have a provision for more complex network structures built from the respective basic cells: the scatternet for Bluetooth, peer-to-peer for UWB, cluster tree or mesh networks for ZigBee, and the ESS for WiFi.

\section{Security}

All the four protocols have the encryption and authentication mechanisms. Bluetooth uses the E0 stream cipher and shared secret with 16-bit cyclic redundancy check (CRC), while UWB and ZigBee adopt the advanced encryption standard (AES) block cipher with counter mode (CTR) and cipher block chaining message authentication code (CBC-MAC), also known as CTR with CBC-MAC (CCM), with 32-bit and 16-bit CRC, respectively.

In 802.11, Wi-Fi uses the RC4 stream cipher for encryption and the CRC-32 checksum for integrity. However, several serious weaknesses were identified by cryptanalysts, any wired equivalent privacy (WEP) key can be cracked with readily available software in two minutes or less, and thus WEP was superseded by Wi-Fi protected access 2 (WPA2), i.e. IEEE 802.11i standard, of which the AES block cipher and CCM are also employed.

\section{E. Transmission Time}

The transmission time depends on the data rate, the message size, and the distance between two nodes. The formula for transmission time $(\mu \mathrm{s})$ can be described as:

$$
T_{\mathrm{tx}}=\left(N_{\text {data }}+\left(N_{\text {data }} / N_{\text {maxPld }} \times N_{\text {ovhd }}\right)\right) \times T_{\text {bit }}+T_{\text {prop }}
$$

where $N_{\text {data }}$ is the data size, $N_{\text {maxPld }}$ is the maximum payload size, $N_{\text {ovhd }}$ is the overhead size, $T_{\text {bit }}$ is the bit time, and $T_{\text {prop }}$ is the propagation time between any two devices. For simplicity, the propagation time is negligible in this paper. The typical parameters of the four wireless protocols used for transmission time evaluation are listed in Table II. Note that the maximum data rate $110 \mathrm{Mbit} / \mathrm{s}$ of UWB is adopted from an unapproved 802.15.3a standard. As shown in Fig. 2, the transmission time for the ZigBee is longer than the others because of the lower data rate $(250 \mathrm{Kbit} / \mathrm{s})$, while UWB requires less transmission time compared with the others. Obviously, the result also shows the required transmission time is proportional to the data payload size and disproportional to the maximum data rate.

TABLE II

TYPICAL SYSTEM PARAMETERS OF THE WIRELESS PROTOCOLS

\begin{tabular}{|l|c|c|c|c|}
\hline \multicolumn{1}{|c|}{ Standard } & Bluetooth & UWB & ZigBee & Wi-Fi \\
\hline \multicolumn{1}{|c|}{ IEEE Spec. } & $\mathbf{8 0 2 . 1 5 . 1}$ & $\mathbf{8 0 2 . 1 5 . 3}$ & $\mathbf{8 0 2 . 1 5 . 4}$ & $\mathbf{8 0 2 . 1 1 a / b / g}$ \\
\hline Max data rate (Mbit/s) & 0.72 & $110^{*}$ & 0.25 & 54 \\
\hline Bit time $(\mu \mathrm{s})$ & 1.39 & 0.009 & 4 & 0.0185 \\
\hline Max data payload (bytes) & $339(\mathrm{DH} 5)$ & 2044 & 102 & 2312 \\
\hline Max overhead (bytes) & $158 / 8$ & 42 & 31 & 58 \\
\hline Coding efficiency $\left.{ }^{+} \%\right)$ & 94.41 & 97.94 & 76.52 & 97.18 \\
\hline * Unapproved 802.15.3a. $^{\text {" Where the data is 10K bytes. }}$ \\
\hline
\end{tabular}

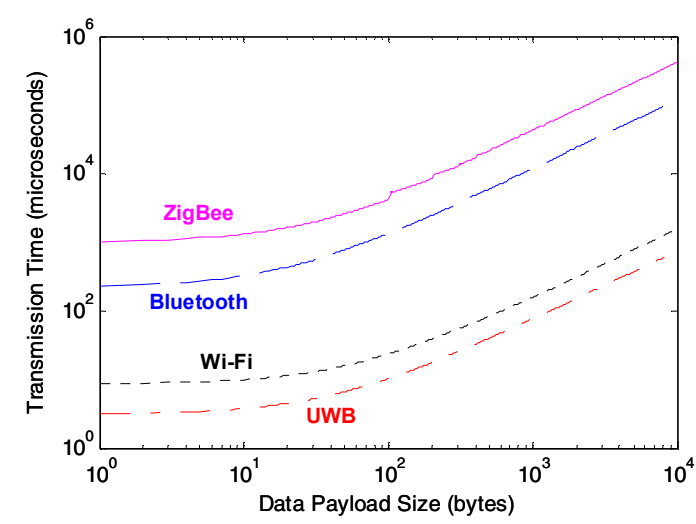

Fig. 2. Comparison of the transmission time versus the data size.

\section{F. Data Coding Efficiency}

In this paper, the data coding efficiency is defined by the ratio of the data size and the message size (i.e. the total number of bytes used to transmit the data). The formula for data coding efficiency (\%) can be described as: 


$$
P_{\text {codeff }}=N_{\text {data }} /\left(N_{\text {data }}+\left(N_{\text {data }} / N_{\text {maxPld }} \times N_{\text {ovhd }}\right)\right)
$$

The parameters listed in Table II are also used for the coding efficiency comparison. Fig. 3 shows the data coding efficiency of the four wireless networks versus the data size. For small data sizes (around smaller than 339 bytes), Bluetooth is the best solution. Also, ZigBee have a good efficiency for data size smaller than 102 bytes. For large data sizes, Bluetooth, UWB, and Wi-Fi have much better efficiency of over $94 \%$, as compared to the $76.52 \%$ of ZigBee (where the data is $10 \mathrm{~K}$ bytes as listed in Table II). The discontinuities in Fig. 2 and 3 are caused by data fragmentation, i.e. the maximum data payload, which is 339, 2044, 102, and 2312 bytes for Bluetooth, UWB, ZigBee, and Wi-Fi, respectively. In a Wi-Fi infrastructure mode, note that most APs connect to existing networks with Ethernet, and therefore limit the payload size to the maximum Ethernet payload size as 1500 bytes. However, for a general comparison, an ad-hoc mode is assumed and the 2312 bytes is adopted in this paper.

For a wireless sensor network in factory automation systems, since most data size of industrial monitoring and control are generally small, (e.g. the temperature data in an environmental monitoring may required less than 4 bytes only), Bluetooth and ZigBee protocols may be a good selection (from a data coding efficiency point of view) in spite of their slow data rate.

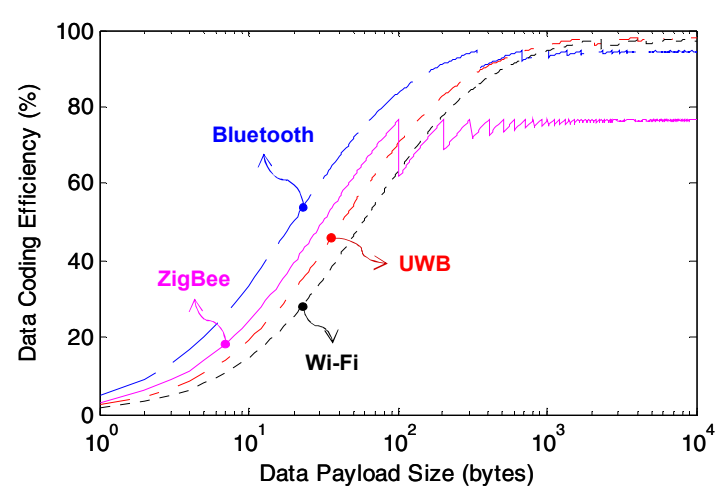

Fig. 3. Comparison of the data coding efficiency versus the data size.

In this section, an evaluation of the Bluetooth, UWB, ZigBee, and $\mathrm{Wi}-\mathrm{Fi}$ on different aspects is provided. It is important to notice that several slight differences exist in the available sources. For example, in the IEEE 802.15.4 standard, the action range is about $10 \mathrm{~m}$, while it is $70-300 \mathrm{~m}$ in the released documents from ZigBee Alliance. Thus, this paper intends to provide information only, since other factors, such as receiver sensitivity and interference, play a major role in affecting the performance in realistic implementations.

\section{Protocol CompleXity AND Power CONSUMPTION}

\section{A. Protocol Complexity}

In this paper, the complexity of each protocol is compared based on the numbers of primitives and events. Table III shows the number of primitives and host controller interface ( $\mathrm{HCI})$ events for Bluetooth, and the numbers of MAC/PHY primitives for UWB, ZigBee, and Wi-Fi protocols. In the $\mathrm{MAC} / \mathrm{PHY}$ layers, the Bluetooth primitives include client service access point (SAP), HCI SAP, synchronous connection-oriented (SCO) SAP, and logical link control and adaptation protocol (L2CAP) primitives. As shown in Fig. 4, the Bluetooth is the most complicated protocol with 188 primitives and events in total. On the other hand, ZigBee is the simplest one with only 48 primitives defined in 802.15.4. This total number of primitives is only about one fourth the number of primitives and events defined in Bluetooth. As compared with the Bluetooth, UWB, and Wi-Fi, the simplicity makes ZigBee very suitable for sensor networking applications due to their limited memory and computational capacity.

TABLE III

NUMBER OF PRIMITIVES AND EVENTS FOR EACH PROTOCOL

\begin{tabular}{|c|c||c|c||c|c|}
\hline Standard & Bluetooth & UWB & ZigBee & Wi-Fi & Standard \\
\hline IEEE Spec. & $\mathbf{8 0 2 . 1 5 . 1}$ & $\mathbf{8 0 2 . 1 5 . 3}$ & $\mathbf{8 0 2 . 1 5 . 4}$ & $\mathbf{8 0 2 . 1 1 a / b / g}$ & IEEE Spec. \\
\hline Primitives & 151 & $77^{*}$ & 35 & 32 & MAC primitives \\
\hline HCl events & 37 & 29 & 13 & 43 & PHY primitives \\
\hline & & & & & "Approved 802.15.3b. \\
\hline
\end{tabular}

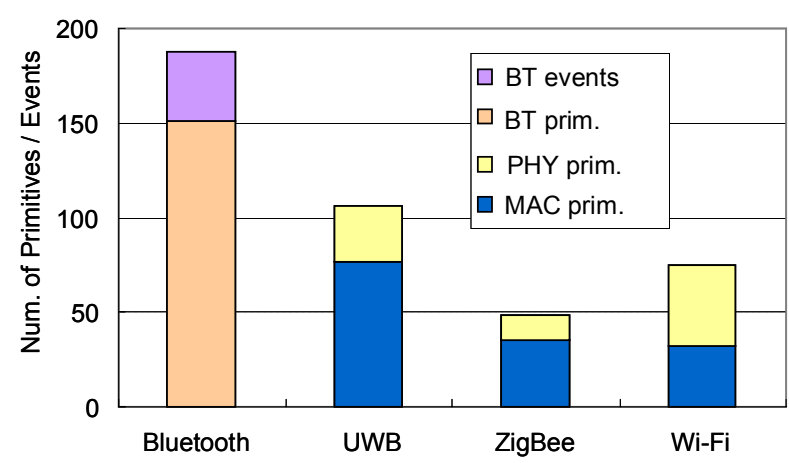

Fig. 4. Comparison of the complexity for each protocol.

\section{B. Power Consumption}

Bluetooth and ZigBee are intended for portable products, short ranges, and limited battery power. Consequently, it offers very low power consumption and, in some cases, will not measurably affect battery life. UWB is proposed for shortrange and high data rate applications. On the other hand, Wi-Fi is designed for a longer connection and supports devices with a substantial power supply. In order to practically compare the power consumption, four wireless products for which detailed characteristics are publicly available are briefly presented as an example, including BlueCore2 [13] from Cambridge Silicon Radio (CSR), XS110 [14] from Freescale, CC2430 [15] from Chipcon of Texas Instruments (TI), and CX53111 [16] from Conexant (previous Intersil's Prism). The current consumptions of the transmit (TX) and receive (RX) conditions for each protocol are shown in Table IV. The data shown are for particular products, although are broadly representative for examples of the same type. Fig. 5 indicates the power consumption in $\mathbf{m W}$ unit for each protocol. Obviously, the 
Bluetooth and ZigBee protocols consume less power as compared with UWB and Wi-Fi. Based on the bit rate, a comparison of normalized energy consumption is provided in Fig. 6. From the $\mathbf{m J} / \mathbf{M b}$ unit point of view, the UWB and $\mathrm{Wi}$ Fi have better efficiency in energy consumption.

In summary, Bluetooth and ZigBee are suitable for low data rate applications with limited battery power (such as mobile devices and battery-operated sensor networks), due to their low power consumption leading to a long lifetime. On the other hand, for high data rate implementations (such as audio/video surveillance systems), UWB and Wi-Fi would be better solutions because of their low normalized energy consumption.

TABLE IV

CURRENT CONSUMPTION OF CHIPSETS FOR EACH PROTOCOL

\begin{tabular}{|c|c|c|c|c|}
\hline Standard & Bluetooth & UWB & ZigBee & Wi-Fi \\
\hline Chipset & BlueCore2 & XS110 & CC2430 & CX53111 \\
\hline VDD (volt) & 1.8 & 3.3 & 3.0 & 3.3 \\
\hline TX (mA) & 57 & $\sim 227.3$ & 24.7 & 219 \\
\hline RX (mA) & 47 & $\sim 227.3$ & 27 & 215 \\
\hline Bit rate $(\mathrm{Mb} / \mathrm{s})$ & 0.72 & 114 & 0.25 & 54 \\
\hline
\end{tabular}

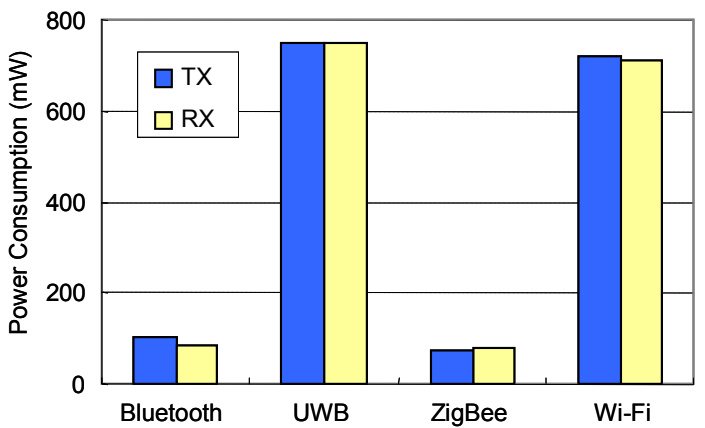

Fig. 5. Comparison of the power consumption for each protocol.

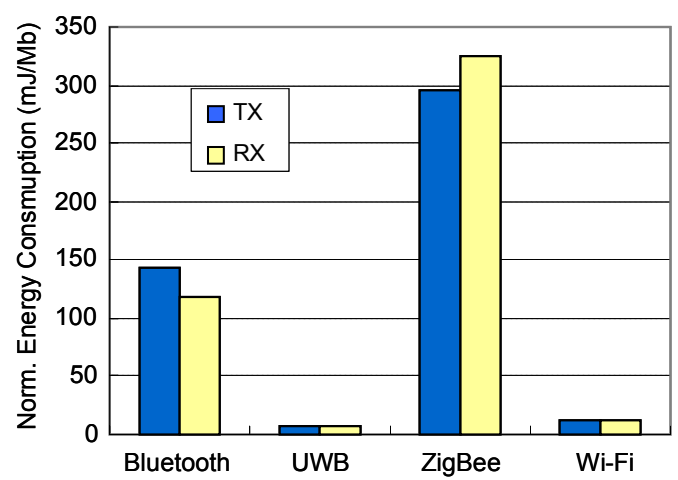

Fig. 6. Comparison of the normalized energy consumption for each protocol.

\section{CONCLUSIONS}

This paper has presented a broad overview of the four most popular wireless standards, Bluetooth, UWB, ZigBee, and WiFi with a quantitative evaluation in terms of the transmission time, data coding efficiency, protocol complexity, and power consumption. Furthermore, the radio channels, coexistence mechanism, network size, and security are also preliminary compared. This paper is not to draw any conclusion regarding which one is superior since the suitability of network protocols is greatly influenced by practical applications, of which many other factors such as the network reliability, roaming capability, recovery mechanism, chipset price, and installation cost need to be considered in the future.

\section{ACKNOWLEDGMENT}

This work was supported by the Ministry of Economic Affairs of Taiwan, ROC, under the Embedded System Software Laboratory in Domestic Communication and Optoelectronics Infrastructure Construction Project.

\section{REFERENCES}

[1] R. Zurawski, "Guest editorial of special section on factory communication systems," IEEE Trans. Ind. Electron., vol. 49, no. 6, pp. 1186-1188, Dec. 2002.

[2] F. L. Lian, J .R. Moyne, and D. M. Tilbury, "Performance evaluation of control networks: Ethernet, ControlNet, and DeviceNet," IEEE Contr. Syst. Mag. vol. 22, no. 1, pp. 66-83, Feb. 2001.

[3] A. Willig, "An architecture for wireless extension of Profibus," in Proc. IEEE Int. Conf. Ind. Electron. (IECON'03), Roanoke, VA, Nov. 2003, pp. 2369-2375.

[4] E. Ferro and F. Potorti, "Bluetooth and Wi-Fi wireless protocols: A survey and a comparison," IEEE Wireless Commun., vol. 12, no. 1, pp. 12-16, Feb. 2005

[5] X. Wang, Y. Ren, J. Zhao, Z. Guo, and R. Yao, "Comparison of IEEE 802.11e and IEEE 802.15.3 MAC," in Proc. IEEE CAS Symp. Emerging Technologies: Mobile \& Wireless Commun, Shanghai, China, May, 2004, pp. 675-680.

[6] Baker, N. "ZigBee and Bluetooth: Strengths and weaknesses for industrial applications," IEE Computing \& Control Engineering, vol. 16, no. 2, pp 20-25, April/May 2005.

[7] D. Porcino and W. Hirt, "Ultra-wideband radio technology: Potential and challenges ahead," IEEE Commun. Mag., vol. 41, no. 7, pp. 66-74, July 2003.

[8] J. S. Lee, "Performance evaluation of IEEE 802.15.4 for low-rate wireless personal area networks," IEEE Trans. Consumer Electron., vol. 52, no. 3, pp. 742-749, Aug. 2006.

[9] J. S. Lee and Y. C. Huang, "ITRI ZBnode: A ZigBee/IEEE 802.15.4 platform for wireless sensor networks," in Proc. IEEE Int. Conf. Systems, Man \& Cybernetics, Taipei, Taiwan, Oct. 2006, pp. 1462-1467.

[10] A. Sikora and V. F. Groza, "Coexistence of IEEE802.15.4 with other systems in the $2.4 \mathrm{GHz}-\mathrm{ISM}$-Band," in Proc. IEEE Instrumentation \& Measurement Technology Conference, Ottawa, May 2005, pp.1786-1791.

[11] K. Shuaib, M. Boulmalf, F. Sallabi, and A. Lakas, "Co-existence of Zigbee and WLAN: A performance study," in Proc. IEEE/IFIP Int. Conf. Wireless \& Optical Communications Networks, Bangalore, India, April 2006.

[12] P. S. Neelakanta and H. Dighe, "Robust factory wireless communications: A performance appraisal of the Bluetooth and the ZigBee collocated on an industrial floor," in Proc. IEEE Int. Conf. Ind. Electron. (IECON'03), Roanoke, VA, Nov. 2003, pp. 2381-2386.

[13] Cambridge Silicon Radio, BlueCore2-External Product Data Sheet. Cambridge, UK, Aug. 2006.

[14] Freescale, XS110 UWB Solution for Media-Rich Wireless Applications. San Diego, CA, Dec. 2004.

[15] Chipcon, CC2430 Preliminary Data Sheet (rev. 1.03). Oslo, Norway, 2006.

[16] Conexant, Single-Chip WLAN Radio CX53111. Newport Beach, CA, 2006. 